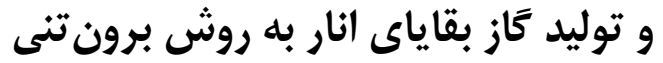

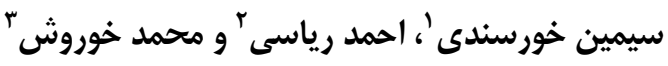

\author{
1- دانشجوى دكترى تغذيه نشخواركنندكان، كروه علوم دامى، دانشكده كشاورزى، دانشكاه صنعتى اصفهان

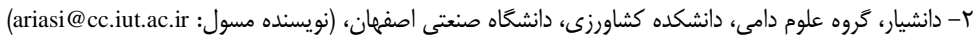

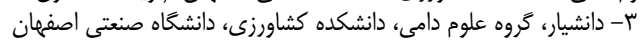

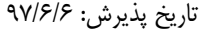

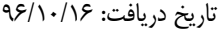

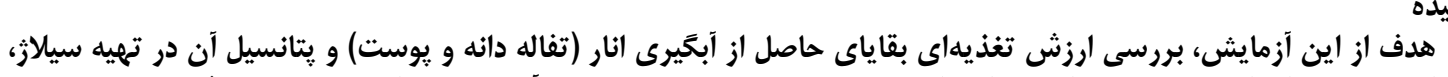

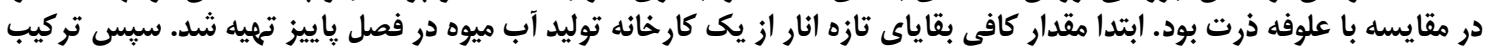

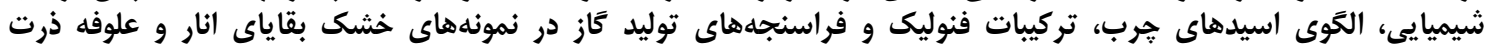

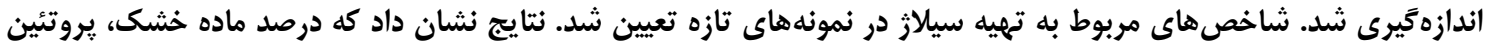

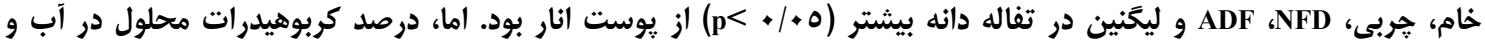

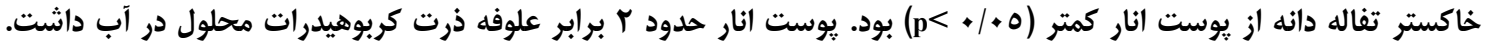

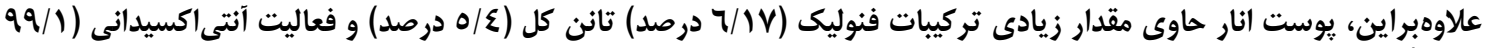

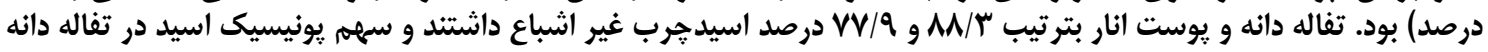

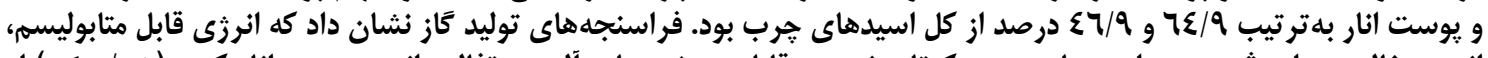

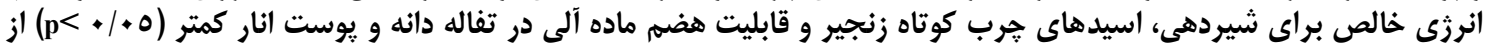

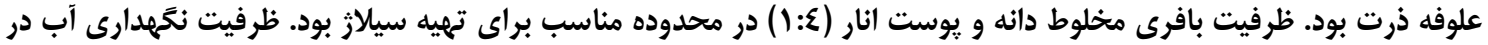

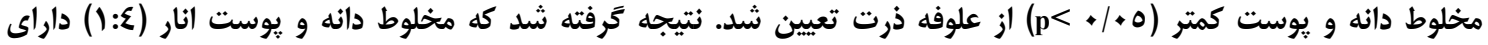

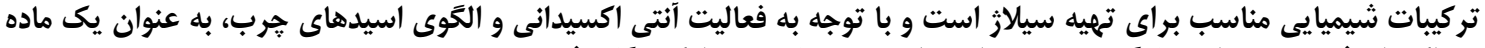

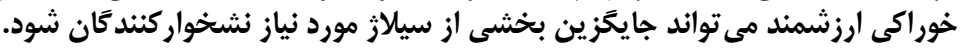

وإزههاى كليدى: محصولات فرعى كثاورزى، تفاله دانه و يوست انار، تركيبات فنوليك، تانن، اسيدهاى جرب ضرورى، فعاليت أنتى اكسيدانى، سيلاز بقاياى انار

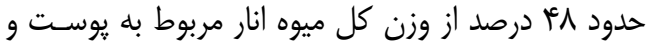

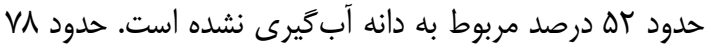

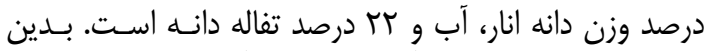

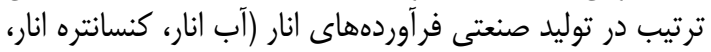

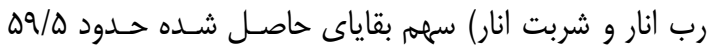

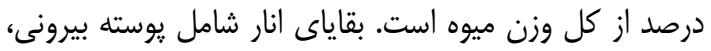

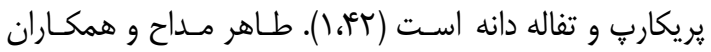

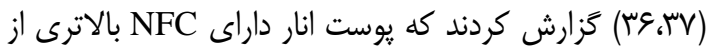

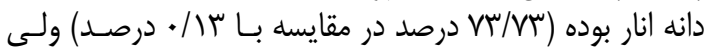

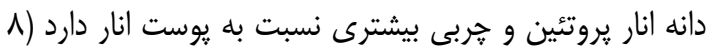

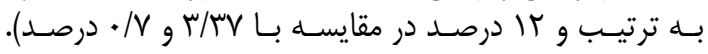

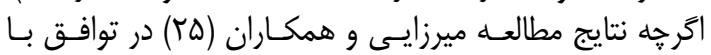

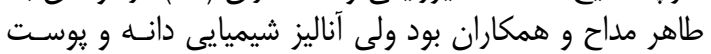

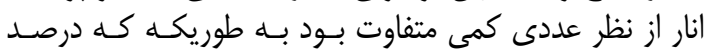

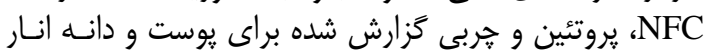

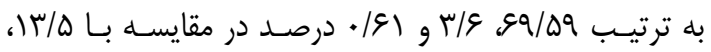

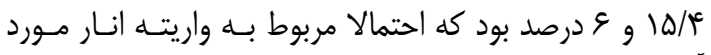

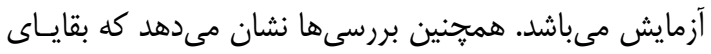

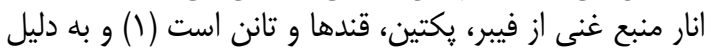

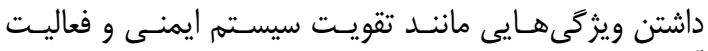

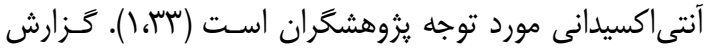

مقدمه

تغيير اقليم و كمبود منابع آب كشاورزى، تهيه خوراك دام

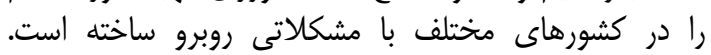

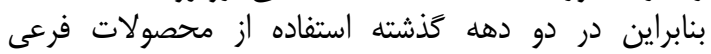

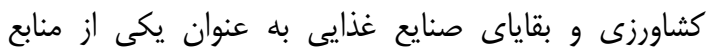

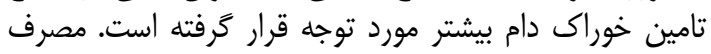

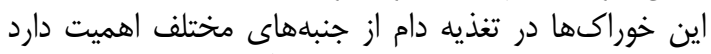

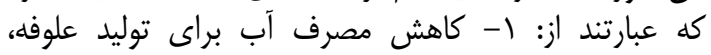

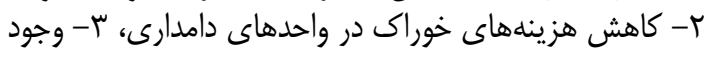

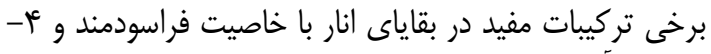

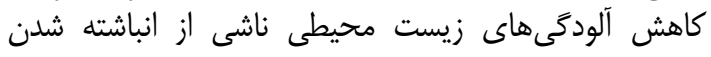

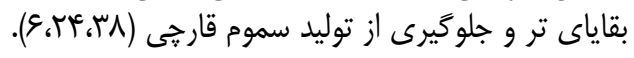

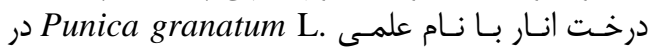

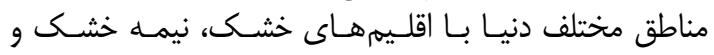

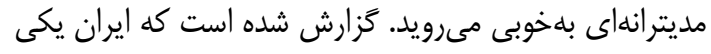

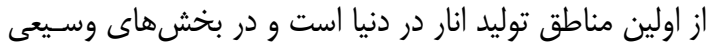

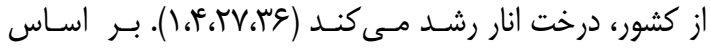

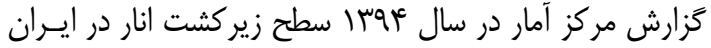

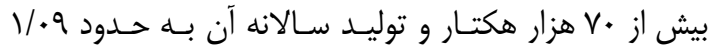

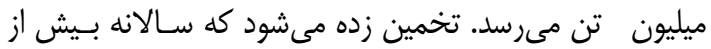

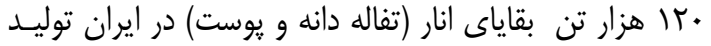

مىشود (1) - (1) - (1). 
ميلىليتر آب مقطر، ه ميلىليتر اسيد سولفوريك خالص و و ماليد

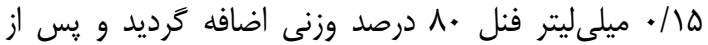

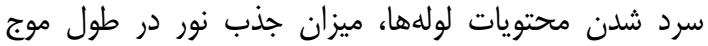

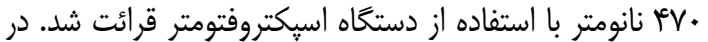

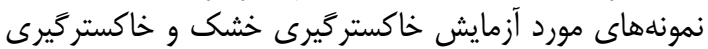

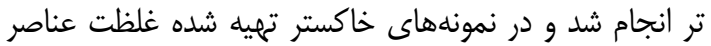

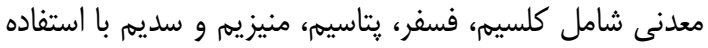

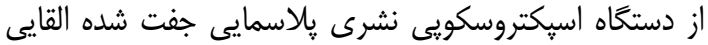
(ICP, Perkin Elmer, Optima 7300 DV, USA) اندازمخيرى شد.

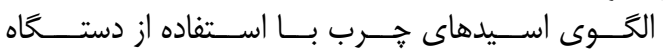

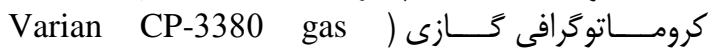
(chromatograph

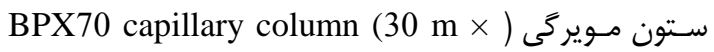
$250 \mu \mathrm{m}$ i.d., $0.32-\mu \mathrm{m}$ phase thickness; Agilent

(Technologies Ireland Ltd.) براى تعيين غلظت كل تركيبات فنوليك و تاننهـا، ابتـــا

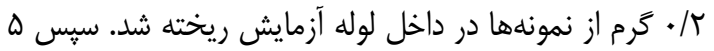

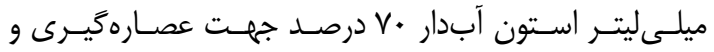

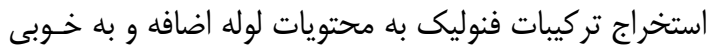

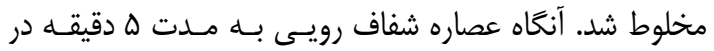

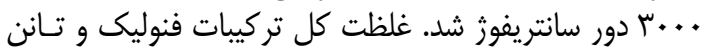

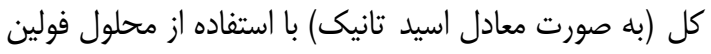

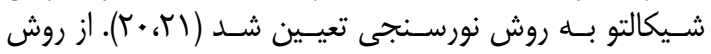

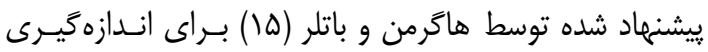

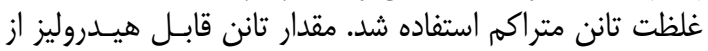

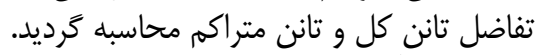

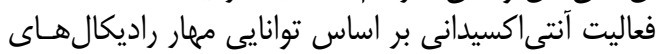
آزاد بـا روش في آتى DPPH (1,1' diphenyl-2-picrylhydrazyl radical, Sigma-Aldrich, USA)

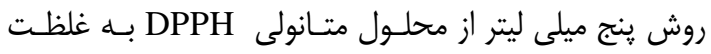

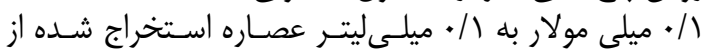

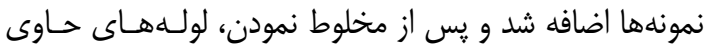

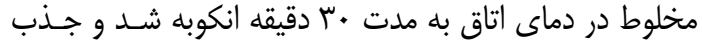

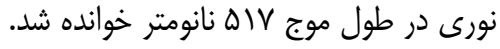

تعيين ارزش تغذيهاى با استفاده از تكنيك توليد كاز

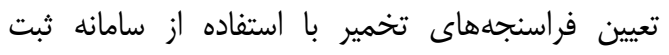

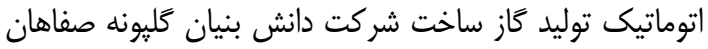

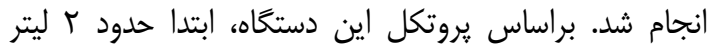

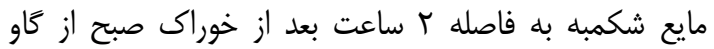

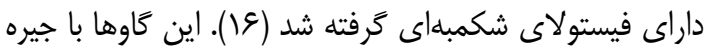

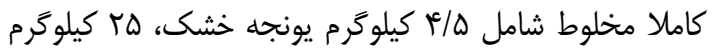

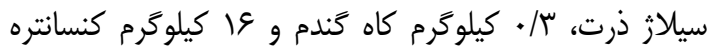

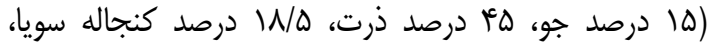

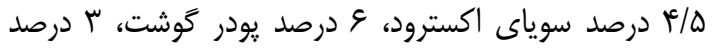

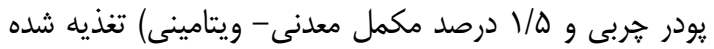

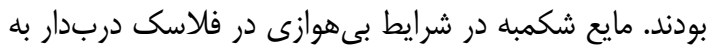

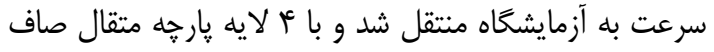

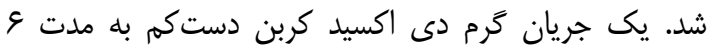
ساعت در محيط كشت تهييه شده براساس بيشنئهاد منك و و

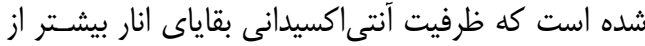

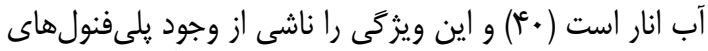

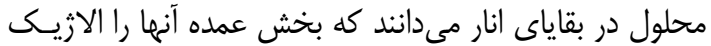

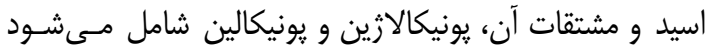

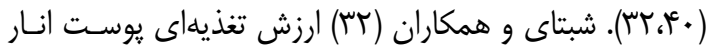

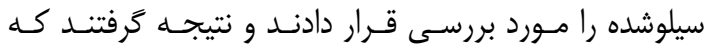

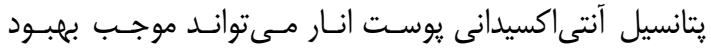

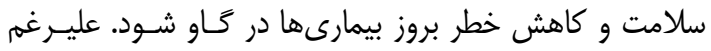

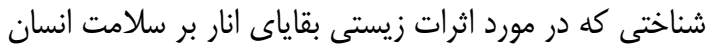

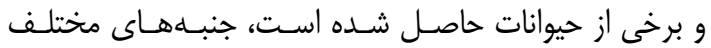

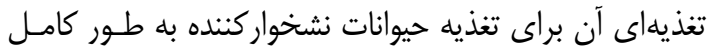

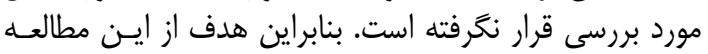

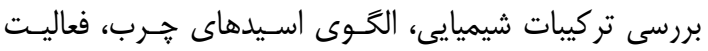

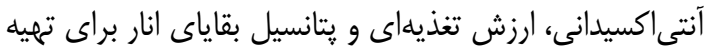
سيلاز در مقايسه با علوفه ذرت بودي

\section{مواد و روشها جمع آورى و آمادهسازى نمونها ها}

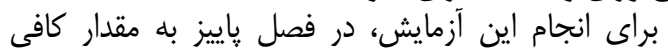

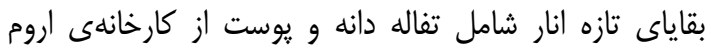

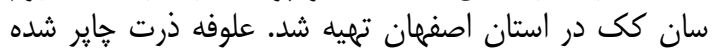

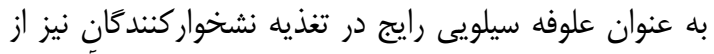

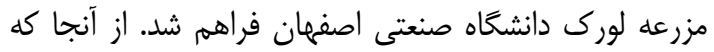

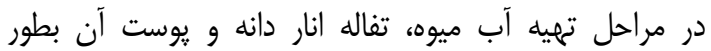

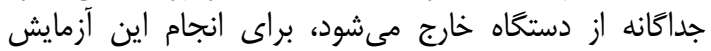

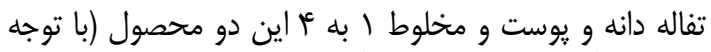
به نسبت آنها در ميوه انار) نيز آماده شد.

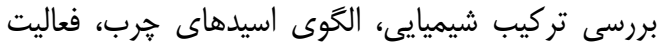

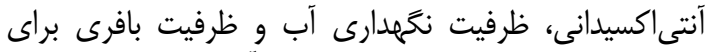

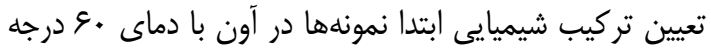

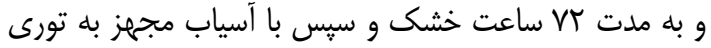

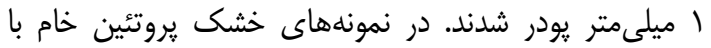

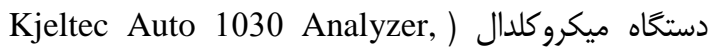
Sweden

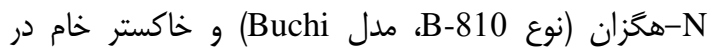

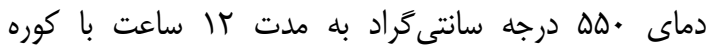
الكتريكى (Shimfan F-47, Iran) اندازهيرى شد شاند (ه).

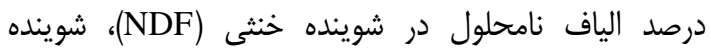
اسيدى (ADF) و ليخنين (ADL) با استفاده از كيسههاى

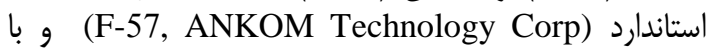

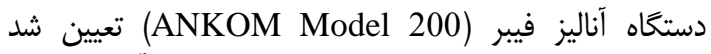

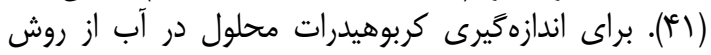

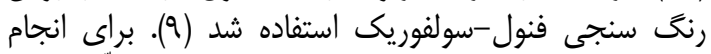

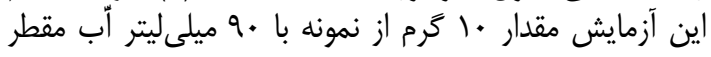

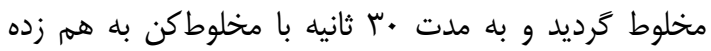

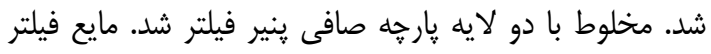

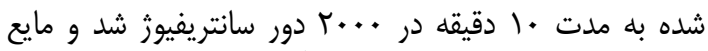

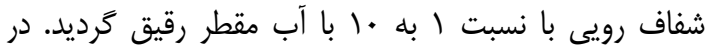
يك لوله آزمايش به يك ميلى ليتر از مايع رقيق شدئ آبه، 


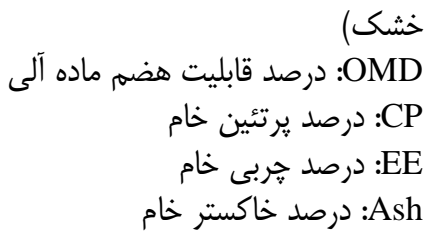
GP

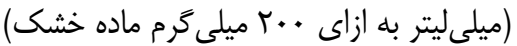

بررسى يتانسيل تهييه سيلاز

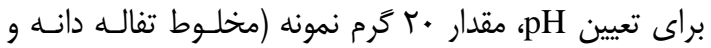

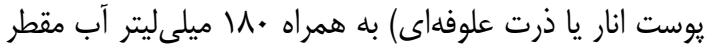

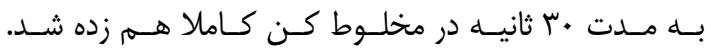

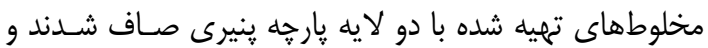

(CRISON Bassic20², EU) pH

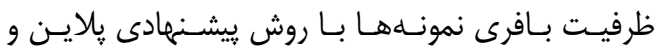

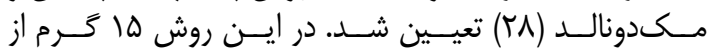

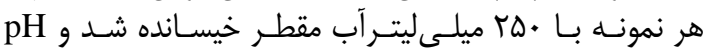

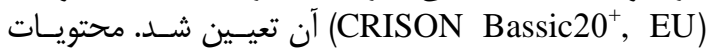

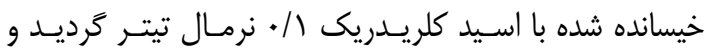

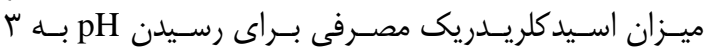

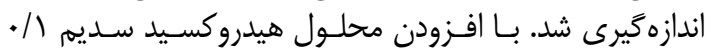

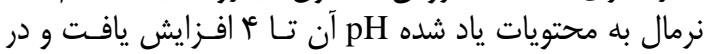

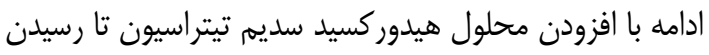

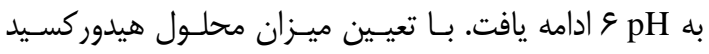

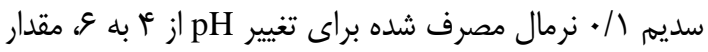

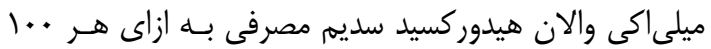

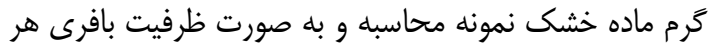
يك از نمونهها بيان گرديد نمونه

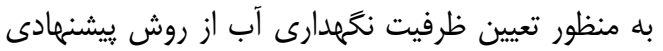

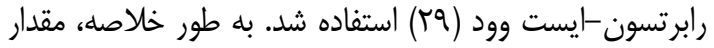

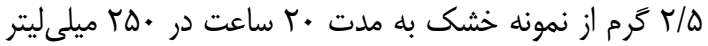

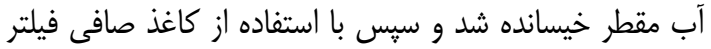

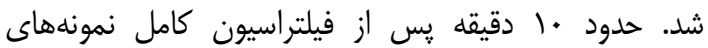

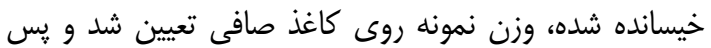

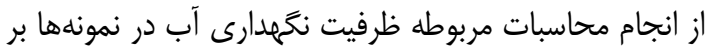

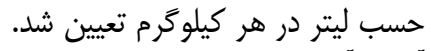

آناليز آمارى دادهن دهر دها

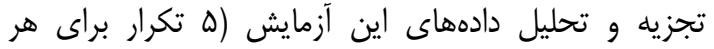

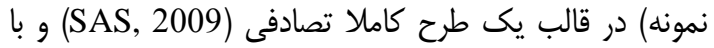

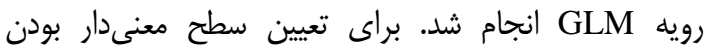

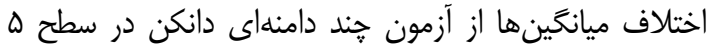

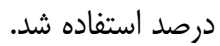
$\mathrm{Y}_{\mathrm{ij}}=\mu+\mathrm{T}_{\mathrm{i}}++\mathrm{e}_{\mathrm{ij}}$

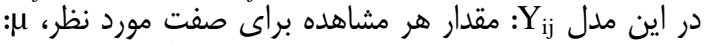

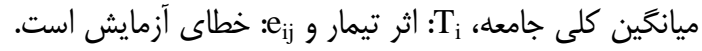

\section{نتايج و بحث تر كيب شيميايى تو بحي}

تركيب شيميايى بقاياى انار (شامل تفاله دانه، يوست و

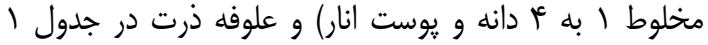
گزارش شده است. نتايج نشان داد كه تفاله دانه انار بيشترين دار دران
استينگايس برقرار شد (ب/)، سپِ مايع شكمبه فيلتر شده به

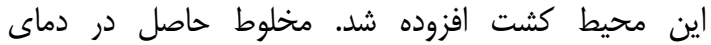

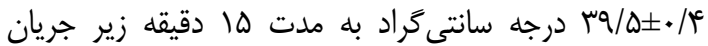

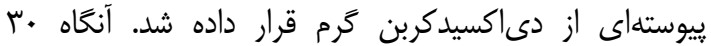

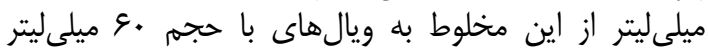

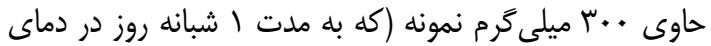
qu درجه سانتى

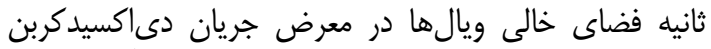

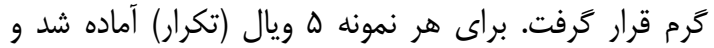

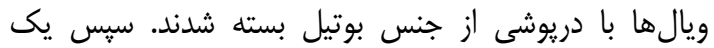

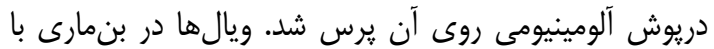

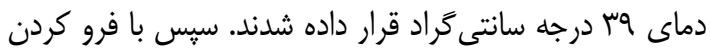

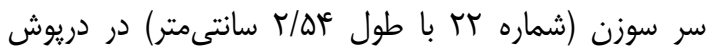

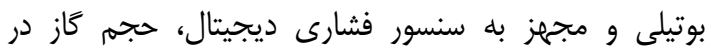

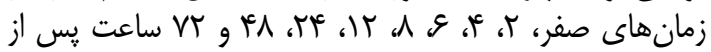

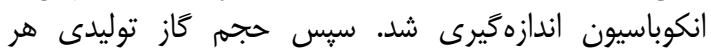

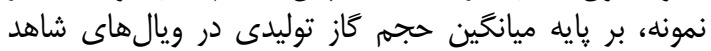

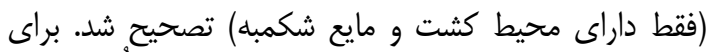

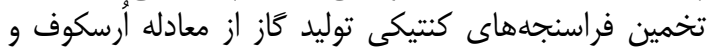
$\mathrm{GP}=\mathrm{A}\left(1-\mathrm{e}^{-\mathrm{c}(\mathrm{T}-\mathrm{L})}\right)$

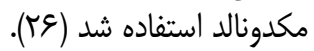
كه در اين معادله: GP حجم كاز توليدى (ميلىليتر در گرم ماده خشك) در زمان A حجم گاز توليدى از بخش قابل تخمير (ميلىليتر به ازاى

هر گرم نمونه)

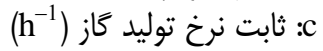

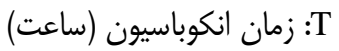
غاخ L غلظت اسيدهاى جرب زان (ساعت) كوتاه زنجير با استفاده از معادله زير SCFA $=\cdot 1 \cdot \mathrm{rmq}^{\mathrm{N}} \times \mathrm{GP}_{24}-\cdot 1 \cdot 9 \cdot 1$ محاسبه شد (IT): كه در اين معادله: ميل SCFA ميلى گرم ماده خشك)

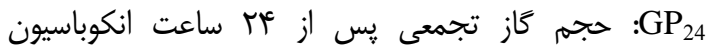

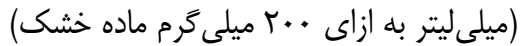

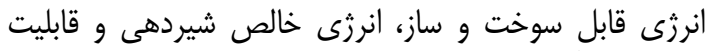

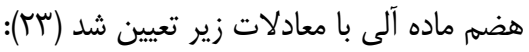

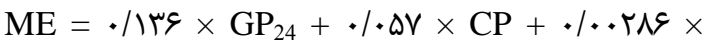
$\mathrm{EE}^{2}+r / r$.

$\left.\mathrm{NE}_{1}=\cdot / 11 \omega \times \mathrm{GP}_{24}+\cdot / \cdot \Delta \mathrm{QV} \times \mathrm{CP}+\cdot / \cdot 1\right)^{\mathrm{F}} \times \mathrm{EE}$ $-\cdot / \cdot \Delta)^{\mathrm{E}} \times \mathrm{Ash}-\cdot / \mathrm{r}$

$\mathrm{OMD}=\cdot / 1 \wedge 9 \times \mathrm{GP}_{24}+\cdot / 44 \wedge \times \mathrm{CP}+.1 .9 \Delta 1 \times$ Ash $+1 \% / \Lambda \Lambda$

كه در اين معادله، ME

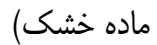
NE 
مخلوط تفاله دانه و يوست انار درصد فسفر كمى داشت. اغلب تئب

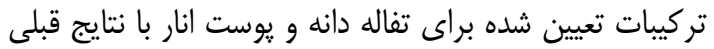

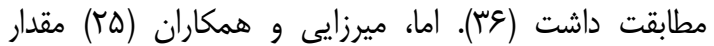

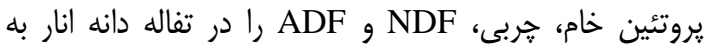

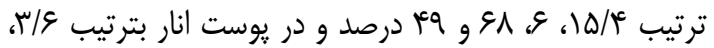

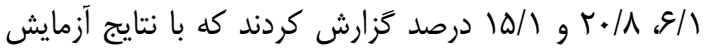

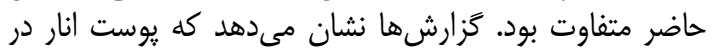

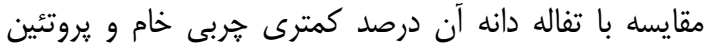

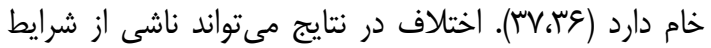

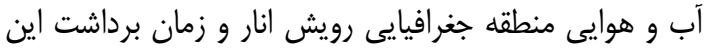

محصول باشد (TV) - (T).

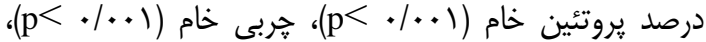

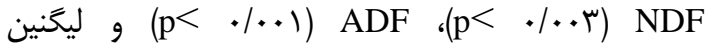

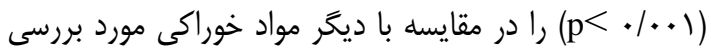

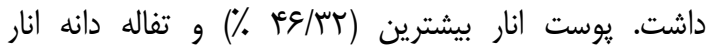

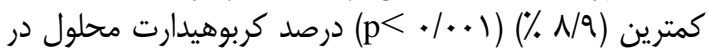

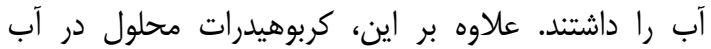

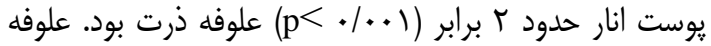

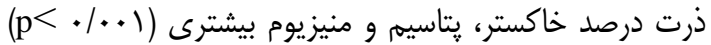

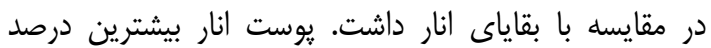

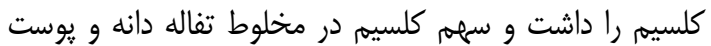

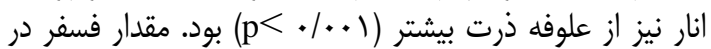

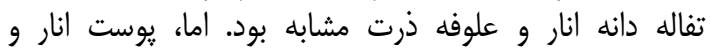

جدول (- ميانگَين تركيب شيميايى بقاياى انار (تفاله دانه، يوست، مخلوط تفاله دانه و يوست) در مقايسه با علوفه ذرت Table 1. Mean chemical composition of pomegranate by-product (seed, peel, mixture of pomegranate seed and peel)

\begin{tabular}{|c|c|c|c|c|c|c|}
\hline $\mathrm{P}$ value & SEM & علوفه ذرت & مخلوط تفاله دانه و & يوست انار & تفاله دانه انار & (درصد از ماده خيميىى) \\
\hline $.1 . .1$ &.$/ 91$. & $r \cdot / \wedge \gamma^{c}$ & $\kappa \Psi / \Gamma \xi^{b}$ & $F r / / T^{b}$ & $\Delta \Psi / \cdot V^{\mathrm{a}}$ & ماده خشك \\
\hline.$/ . .1$ & .1 .91 & $s / \psi^{b}$ & $4 / .9^{c}$ & $r / q \cdot{ }^{d}$ & $q / 9 \vee^{a}$ & يروتئين خام \\
\hline.$/ . .1$ &.$/ T \Lambda$. & $r / g Y^{c}$ & $r / v q^{b}$ & $1 / 9 r^{c}$ & $|r / \cdot|^{\mathrm{a}}$ & هربى خام \\
\hline.$/ . .1$ &.$/ . \Delta F$ & $N / \cdot^{\mathrm{a}}$ & r/F & $r / \wedge q^{b}$ & $r / \cdot r^{\mathrm{c}}$ & خاكستر \\
\hline.$/ . .1$ & $. / 4 \times q$ & $r / r^{c}{ }^{c}$ & $r V / A^{c} \Lambda^{b}$ & $r g / r r^{a}$ & $N / q \cdot{ }^{d}$ & كربوهيدرات محلول در آب \\
\hline ...r & $\cdot / V \cdot 9$ & $\Delta \backslash / \Gamma \omega^{b}$ & $r \cdot / \cdot v^{c}$ & $r M / \mu^{4} e^{d}$ & $\Delta Q / r^{a}$ & NDF \\
\hline $.1 . .1$ & . IVAV & $r r / v q^{b}$ & $19 / \Delta \mu^{c}$ & $\mid w / e^{d}$ & $r N / q \Lambda^{a}$ & $\mathrm{ADF}$ \\
\hline.$/ . .1$ & g & $\Delta / \cdot V^{c}$ & $q / \cdot r^{b}$ & $\Delta / / \Lambda^{C}$ & $r r / r r^{a}$ & ليحَنين \\
\hline.$/ . .1$ &.$|\cdot r|$ & $\cdot / \mu \cdot^{c}$ &.$/ \mathrm{ma}^{\mathrm{b}}$ &.$/ 4 c^{\mathrm{a}}$ & $\cdot / 1 \Delta^{\mathrm{d}}$ & كلسيم \\
\hline.$/ . .1$ &.$/ .+r$ &.$/ r \Lambda^{\mathrm{a}}$ &.$/ 1 r^{b}$ & $\cdot / \cdot^{c}$ & $\cdot / r v^{a}$ & فسفر \\
\hline.$/ . .1$ & $\cdot / \cdot r$ & $1 / \pi r^{a}$ & $1 / 11^{c}$ & $1 / r \Delta^{b}$ & $\cdot / \Delta \Delta^{\mathrm{d}}$ & يتاسيم \\
\hline $.1 . .1$ & $.1 \cdot r$. & $\cdot / r \cdot{ }^{a}$ & $.1 .9^{\mathrm{b}}$ & $\cdot 1 \cdot 0^{\mathrm{b}}$ & $.11 \cdot^{\mathrm{b}}$ & 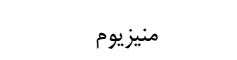 \\
\hline.$/ \cdot r$ & . & $\cdot / \cdot 1^{\mathrm{c}}$ & $\cdot / \Lambda^{\mathrm{a}}$ & $\cdot / r \cdot{ }^{a}$ & $\cdot / \cdot^{\mathrm{b}}$ & سديم \\
\hline
\end{tabular}

لينولئيك اسيد و اولئيك اسيد بود. نسبت مجموع اسيدهاى

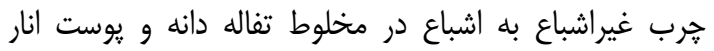

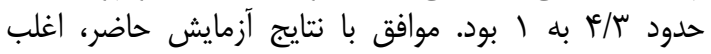

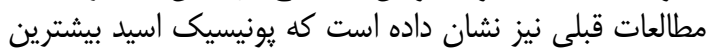

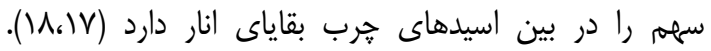

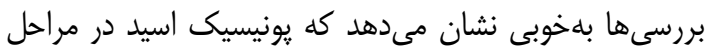

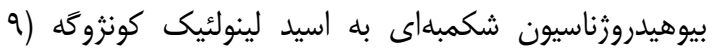

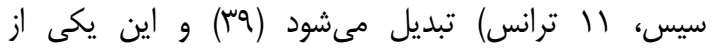

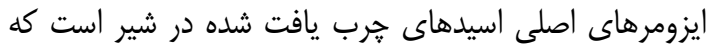

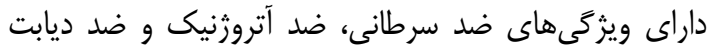

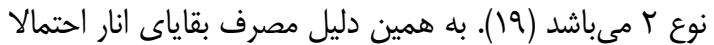

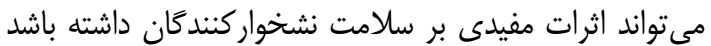

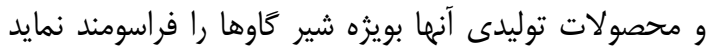

\section{الخوى اسيدهاى جرب إنوب}

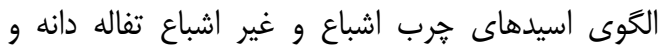

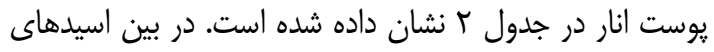

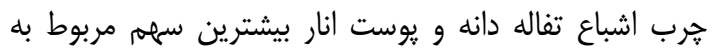

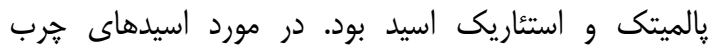

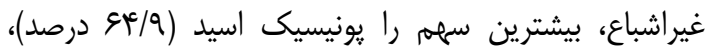

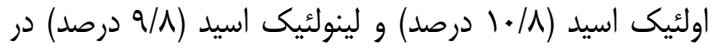

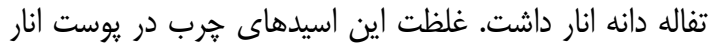

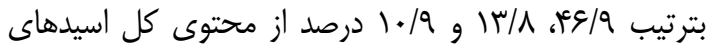

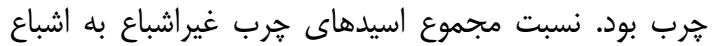

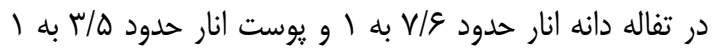

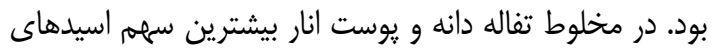

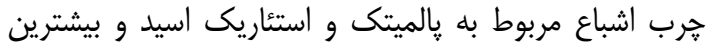

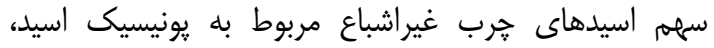


جدول r- مقايسه الخُى اسيدهاى جرب موجود در بقاياى انار (تفاله دانه، يوست، مخلوط دانه و يوست) Table 2. Comparison of the fatty acid profile of pomegranate by-product (seed, peel, mixture of pomegranate seed and peel)

\begin{tabular}{|c|c|c|c|}
\hline مخلوط تفاله دانه و يوست انار' & يوست انار & تفاله دانه انار & الكَى اسيدهاى خرب (درصد) \\
\hline.$/$. &.$/ \cdots$ &.$/$. & لوريك اسيد (C12:0) \\
\hline$\cdot / 0$ & .19 & $\cdot / 1$ & مريستيك اسيد (C14:0) \\
\hline$\cdot / 48$ & $\cdot / \mu \cdot$ & $\cdot / 1$ & يالميتوليك اسيد (C16:1, 9C) \\
\hline$V / V f^{c}$ & $\Lambda / \kappa^{\leftarrow}$ & $\Delta / 1$. & استئاريك اسيد (C18:0) \\
\hline$\cdot / 48$ & $\cdot / \Delta$ & $\cdot / \mu$ & كانجوگيت لينولئيك اسيد (C18:2, 11t, 15c) \\
\hline $1 \cdot 191$ & $1 \cdot / 9$ & $9 / \wedge$. & لينولئيك اسيد (C18:2, 9c, 12c) \\
\hline$\cdot / V R$ & $\cdot / v \cdot$ & $\cdot / \Lambda$ & كاما لينولنيك اسيد (C18:3, 6c, 9c, 12c) \\
\hline $1 / \Delta \Lambda$ & $1 / \mathrm{r}$ & $1 / 1$ & آلفا لينولنيك اسيد (C18:3, 9c, 12c, 15c) \\
\hline$\Delta \cdot / \Delta \cdot$ & $+\varepsilon / q$. & $94 / q$. & يونيسيك اسيد (C18:3, 9c, 11t, 13c) \\
\hline$\cdot / \mathrm{r \Lambda}$ & $\cdot / \mu$ & $\cdot / 4$ & اروسيك اسيد (C22:1, 13c) \\
\hline$r \cdot / \cdot r$ & $r r / 1$. & $11 / \mathrm{V}$ & كل اسيدهاى خرب اشباع \\
\hline $\mathrm{V} / \mathrm{qu}$ & $\mathrm{VV} / \mathrm{q}$. & $M / \mu$. & كل اسيدهاى خرب غير اشباع \\
\hline 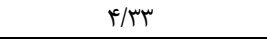 & T/QT & $V / \Delta \Delta$ & نسبت كل اسيدهاى خرب غير اشباع به اشباع \\
\hline
\end{tabular}

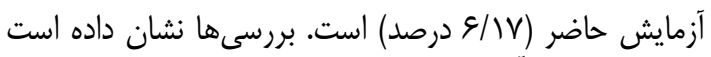

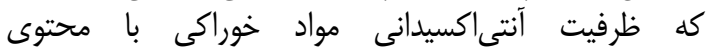

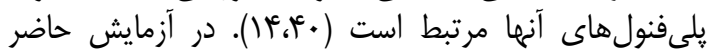

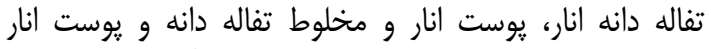

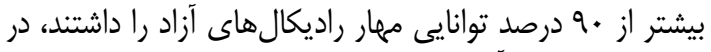

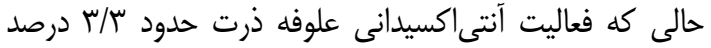

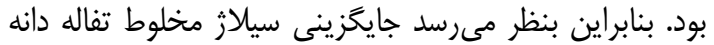

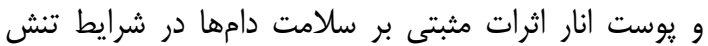

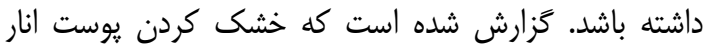

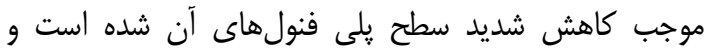

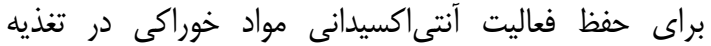

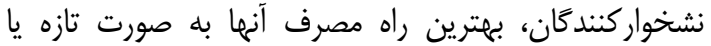

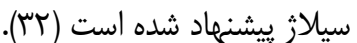

$$
\text { فعاليت آنتى اكسيدانى }
$$

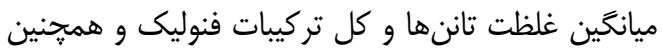

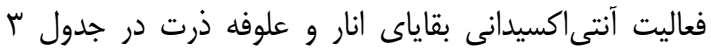

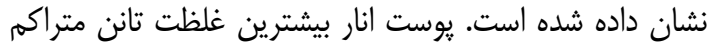

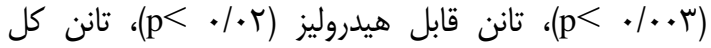

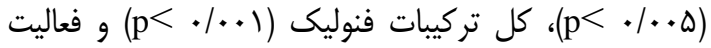

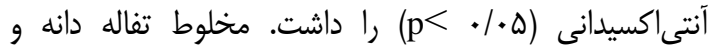

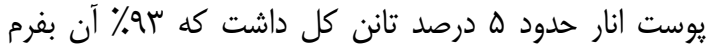

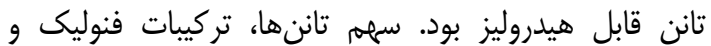

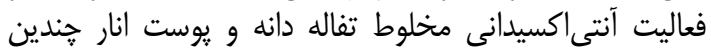

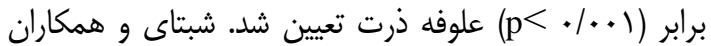

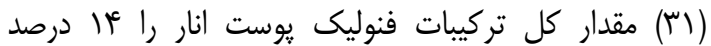

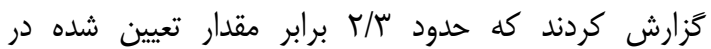

جدول ب- ميانگين تاننها، كل تركيبات فنوليك و فعاليت آنتىاكسيدانى بقاياى انار (تفاله دانه، يوست، مخلوط تفاله دانه و يوست) در مقايسه با علوفه ذرت

Table 3. Mean tannins, total phenolic compounds and antioxidant activity of pomegranate by-product (seed, peel, mixture of pomegranate seed and peel) compare to corn forage.

\begin{tabular}{|c|c|c|c|c|c|c|}
\hline$P$ value & SEM & علوفه ذرت & مخلوط تفاله دانه & يوست انار & تفاله دانه انار & \\
\hline.$/+r$ & $.1+9$ & $\cdot 1 \cdot r^{\mathrm{d}}$ & $\cdot / \Gamma \Delta^{\mathrm{b}}$ &.$/ 44^{a}$ &.$/ 11^{\mathrm{c}}$ & تانن متراكم (درصد از ماده خشك) \\
\hline$\cdot / \cdot r$ & 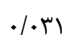 & $\cdot 1 \cdot 9^{d}$ & $f / \& q^{b}$ & $r / q \varphi^{a}$ & $1 / T \Lambda^{\mathrm{c}}$ & تانن قابل هيدروليز (درصد از ماده خشك) \\
\hline$\cdot 1 \cdots \Delta$ &.$/ \cdot \mathrm{rV}$ &.$/ 11^{\mathrm{d}}$ & $\Delta / \cdot 1^{\mathrm{b}}$ & $\Delta / \kappa^{c}{ }^{a}$ & $1 / q^{c}$ & تانن كل(درصد از ماده خشك) \\
\hline$\cdot 1+\cdot 1$ & $\cdot / 1 \Lambda$ & $\cdot / f^{d}$ & $\Delta / \bar{c} \cdot^{b}$ & $q / 1 v^{a}$ & $|/ 9|^{\mathrm{c}}$ & كل تركيبات فنوليك (درصد از ماده خشك) \\
\hline$\cdot 1 \cdot \Delta$ & 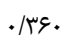 & derd & $q V / \Delta V^{b}$ & ११/।. ${ }^{a}$ & $9) / \uparrow 9^{c}$ & فعاليت آنتى اكسيدانى ' (درصد) \\
\hline
\end{tabular}

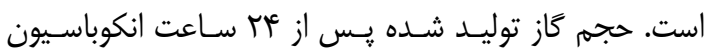

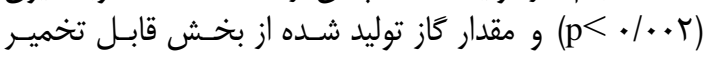

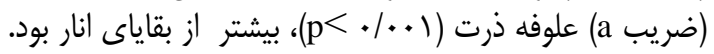

ارزش تغذيهاى با تكنيك توليد كاز

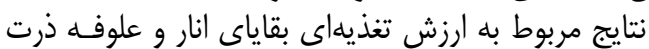

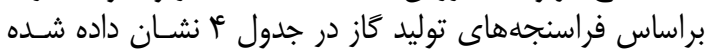




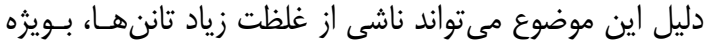

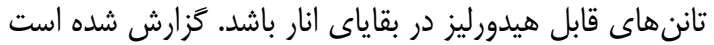

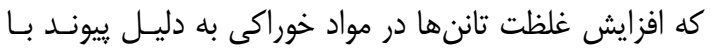

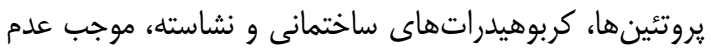

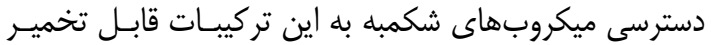

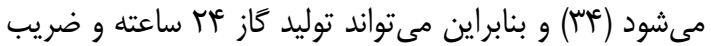

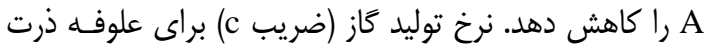

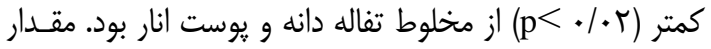

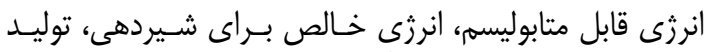

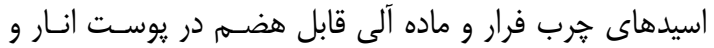

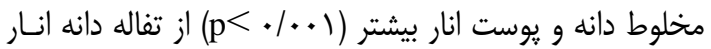

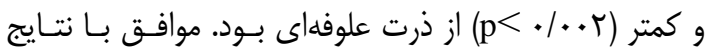

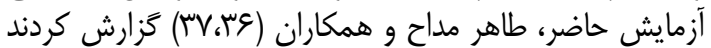

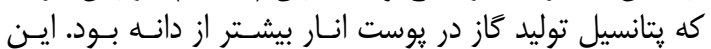

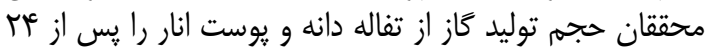

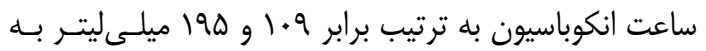

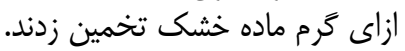

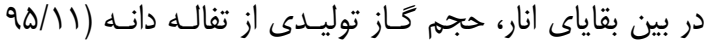

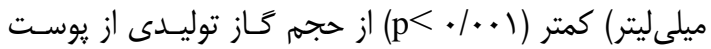

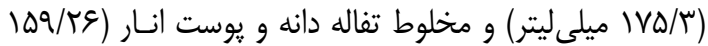

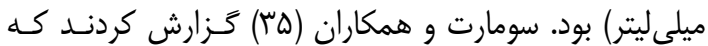

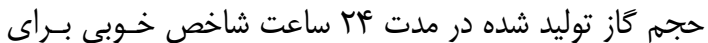

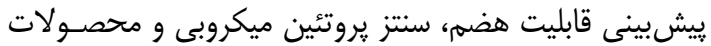

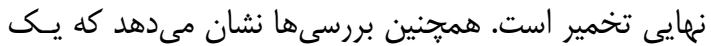

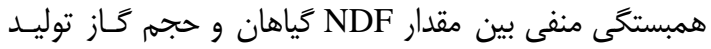

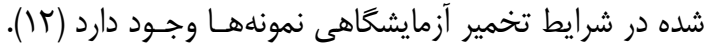

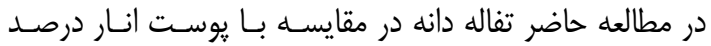

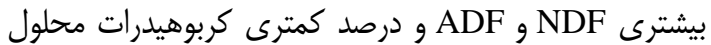

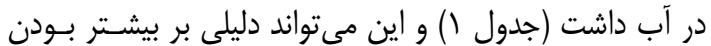

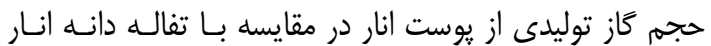

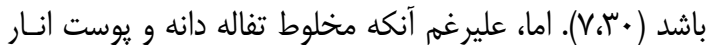

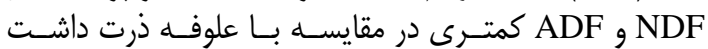

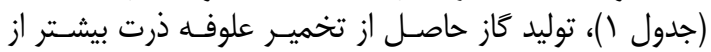

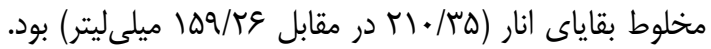

جدول عا- ميانگين فراسنجههاى توليد كاز بقاياى انار (تفاله دانه، يوست، مخلوط تفاله دانه و يوست) در مقايسه با علوفه ذرت Table 4. Mean gas production parameters of pomegranate by-product (seed, peel, mixture of pomegranate seed and peel) compare to corn forage

\begin{tabular}{|c|c|c|c|c|c|c|}
\hline$P$ value & SEM & علوفه ذرت & مخلوطت تفاله دانه & 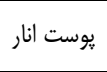 & تفاله دانه انار & فراسنجههاى توليد كَاز \\
\hline.$/ .+4$ & $8 / \Delta 9 \Delta$ & $r 1 . / r \omega^{a}$ & $109 / r q^{6}$ & $I V D / \mu^{b}$ & $9 \Delta / 11^{c}$ & حجم كاز توليد شده در عاب ساعت \\
\hline.$/ \cdots 1$ & $9 /$ TqF & $r \Delta S / Q r^{\mathrm{a}}$ & $\Lambda \Lambda \Delta / r \Delta^{b}$ & $r \cdot F / F \theta^{b}$ & $1 \cdot N / \kappa^{2} e^{C}$ & ضريب A r \\
\hline$\cdot / \cdot f^{f}$ &.$/ \varphi^{c}$ & $.1 .99^{\mathrm{b}}$ & $\cdot 1 \cdot \wedge^{\mathrm{a}}$ & $\cdot / \cdot \mathrm{vA}^{\mathrm{ab}}$ & $\cdot / \cdot \wedge \mathrm{r}^{\mathrm{a}}$ & ضريب c' \\
\hline.$/ .+1$ &.$/ I V \Lambda$ & $N / r^{a}{ }^{a}$ & $s|\Lambda|^{\mathrm{b}}$ & $v / \backslash \varepsilon^{\mathrm{b}}$ & $\Delta / 9 c^{c}$ & انرزى قابل متابوليسهْه \\
\hline.$/ \cdots 1$ & . /NQT & $c / \omega \cdot{ }^{a}$ & - l & $r / 99^{b}$ & $r / \cdot F^{c}$ & انرزى خالص براى شيردهى \\
\hline $.1 . .1$ & $1.1 \% q$ & $\cdot / q r^{\mathrm{a}}$ &.$/ v *^{b}$ &.$/ w^{b}$ &.$/ F T^{C}$ & توليد اسيدهاى هرب كوتاه زنجير' \\
\hline $.1 \cdot 1$ & I/ISV & 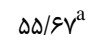 & $r \Delta / Y^{C b}$ & $\mathrm{FV} / \mathrm{c} \cdot \mathrm{o}^{\mathrm{b}}$ & $r q / 4)^{c}$ & قابليت هضم ماده آلىى \\
\hline \multicolumn{7}{|c|}{ 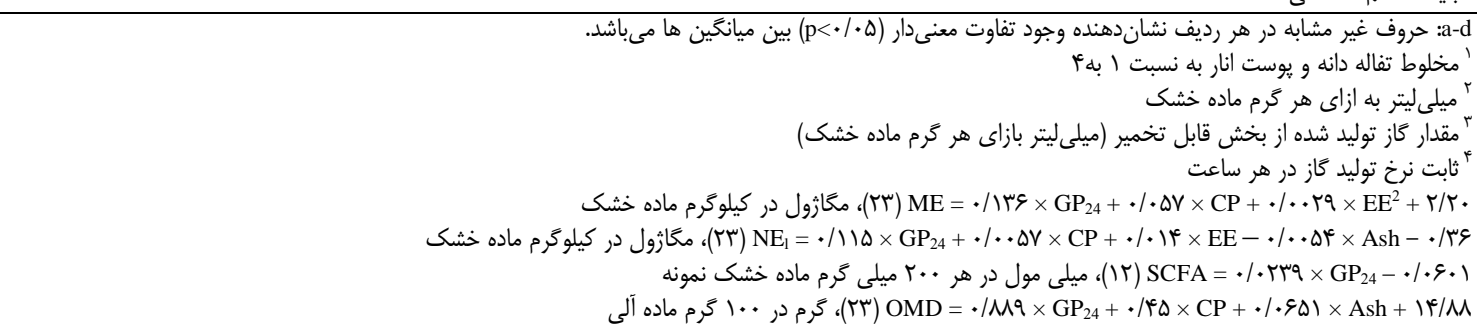 } \\
\hline
\end{tabular}

(حدود •9 ميلى اكى الان) خيلى كمتر است. ظرفيت بافرى

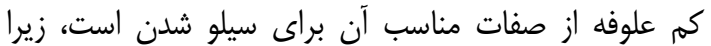

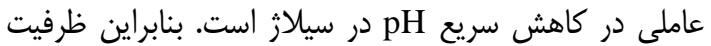

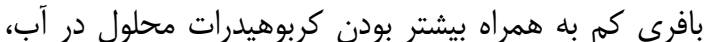

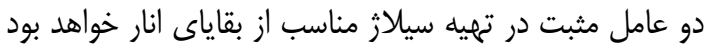

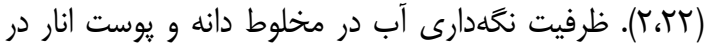

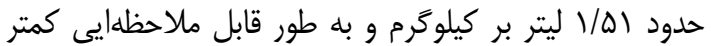

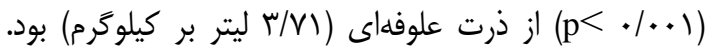

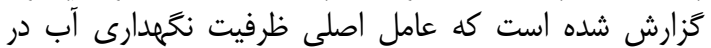

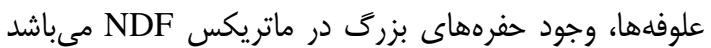

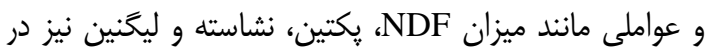

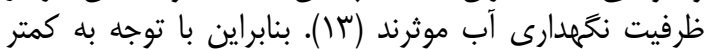

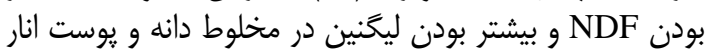

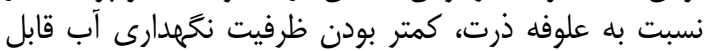

قابليت تهيه سيلاز با توجه به نتايج مربوط به كريه كربوهيدرات محلول در آب،

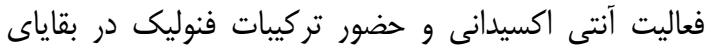

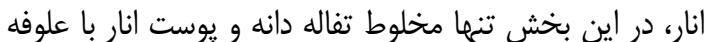

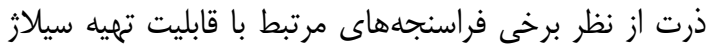

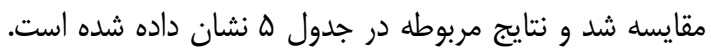
pH

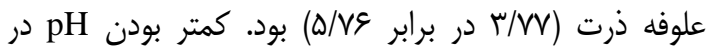
بقاياى انار را مىتوان با مقادير نسبتا زياد تانن قابل دابل هيدروليز

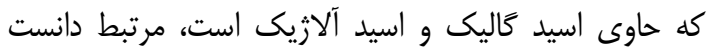

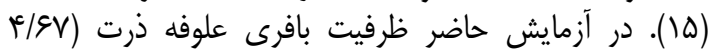

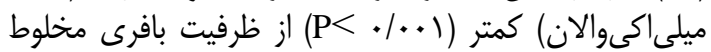

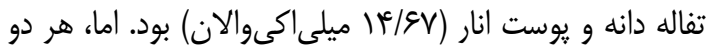

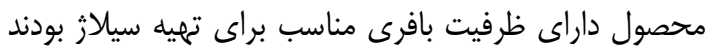

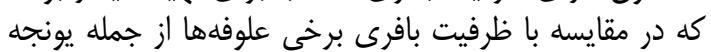


جدول ه- ميانگين طH، ظرفيت بافرى و ظرفيت نخهدارى آب در بقاياى انار (تفاله دانه، يوست، مخلوط تفاله دانه و يوست) در مقايسه با علوفه ذرت Table 5. Mean pH, buffering capacity and water storage capacity of pomegranate by product (seed, peel, mixture of pomegranate seed and peel) compare to corn forage

\begin{tabular}{|c|c|c|c|c|}
\hline 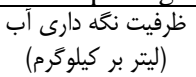 & ظرفيت بافرى (meq) & $\mathrm{HCL}^{\prime}$ (meq) & $\mathrm{pH}$ & فراسنجه ها \\
\hline $1 / \Delta \cdot^{D}$ & $1 f / q V^{a}$ & (11/ & $\mathrm{r} / \mathrm{VV}^{\mathrm{D}}$ & مخلوط تفاله دانه و يوست انار \\
\hline$r / V r^{a}$ & $r / 9 V^{\circ}$ & $\mid V / r r^{a}$ & $\Delta / V \varepsilon^{\mathrm{a}}$ & علوفه ذرت \\
\hline $.1 \cdot r$. & $\cdot / * r$ & 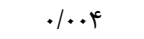 & . $1 \cdot$ th & SEM \\
\hline$\cdot 1+.1$ & $.1 \cdots 1$ & $\cdot 1+.1$ & $\cdot 1+\cdot 1$ & $P$ value \\
\hline
\end{tabular}

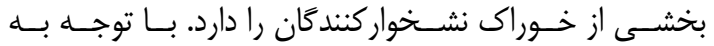

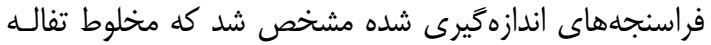

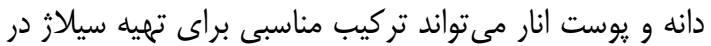

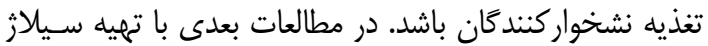

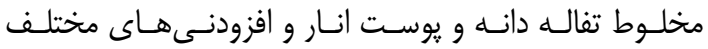

اطلاعات دقيقترى به دست خواهد آمد.

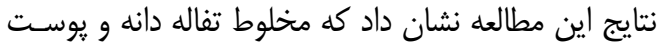

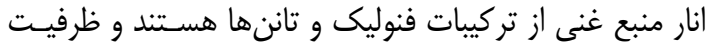

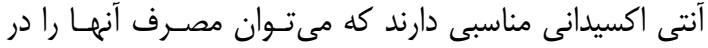

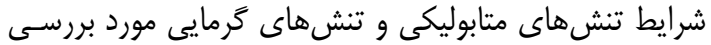

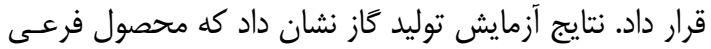

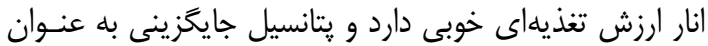

منابع

1. Abbasi, H., K. Rezaei and L. Rashidi. 2008. Extraction of essential oils from the seeds of pomegranate using organic solvents and supercritical $\mathrm{CO}_{2}$. Journal of the American Oil Chemists' Society, 85: 8389

2. Addah, W., J. Baah, P. Groenewegen, E.K. Okine and T.A. McAllister. 2011. Comparison of the fermentation characteristics, aerobic stability and nutritive value of barley and corn silages ensiled with or without a mixed bacterial inoculant. Journal of Animal Science, 91: 133-146.

3. Adesogan, A.T., N.M. Krueger, B. Salawu, D.B. Dean and C.R. Staples. 2004. The Influence of treatment with dual purpose bacterial inoculants or soluble carbohydrates on the fermentation and aerobic stability of Bermudagrass. Journal of Dairy Science, 87: 3407-3416.

4. Aviram, M., M. Rosenblat, D. Gaitini, S. Nitecki, A. Hoffman, L. Dornfeld, N. Volkova, D. Presser, J. Attias and H. Liker. 2004. Pomegranate juice consumption for 3 years by patients with carotid artery stenosis reduces common carotid intima media thickness, blood pressure and LDL oxidation. Clinical Nutrition, 23: 423-433.

5. AOAC. 2000. Official methods of analysis. $7^{\text {th }}$ ed., Official Methods of Analysis of AOAC International, Gaithersburg, M.D, USA.

6. Bampidis, V. A. and P. H. Robinson. 2006. Citrus by products as ruminant feeds: A review. Animal Feed Science and Technology, 128: 175-217.

7. Blummel, M. and K. Becker. 1997. The degradability characteristics of fifty-four roughages and roughage neutral detergent fibres as described by in vitro gas production and their relationship to voluntary feed intake. British Journal of Nutrition, 77: 757-768.

8. Brand Williams, W., M. Cuvelier and C. Berset. 1995. Use of a free radical method to evaluate antioxidant activity. LWT-Food Science and Technology, 28: 25-30.

9. Dubois, M., K.A. Gilles, J.K. Hamilton, P. Rebers and F. Smith. 1956. Colorimetric method for determination of sugars and related substances. Analytical Chemistry, 28: 350-356.

10. Feizi, R., A. Ghodratnama, M. Zahedifar, M.D. Mesgaran and M. Raisianzadeh. 2010. Apparent digestibility of pomegranate seed fed to sheep. ADSA®-PSA-AMPA-CSAS-ASAS Joint Annual Meeting, P:194, Denever, Colorado.

11. Feizi, M., M. Zahedifar, M. Danesh Mesgaran, M. Raisianzadeh and V. Kashki. 2010. Effects of urea treatment on total contents and in vitro gas production of ensiled pomegranate peel. The $4{ }^{\text {th }}$ Congress on Animal Science, P: 2296, Karaj, Iran.

12. Gatachew, G., H. Makkar and K. Becker. 2002. Tropical browse: contents of phenolic compounds, in vitro gas production and stoichiometric relationship between short chain fatty acid and in vitro gas production. The Journal of Agricultural Science, 139: 341-352.

13. Giger Reverdin, S. 2000. Characterisation of feedstuffs for ruminants using some physical parameters. Animal Feed Science and Technology, 86: 53-69.

14. Gil, M.I., F.A. Tomas Barberan, B. Hess-Pierce, D.M. Holcroft and A.A. Kader. 2000. Antioxidant activity of pomegranate juice and its relationship with phenolic composition and processing. Journal of Agricultural and Food Chemistrv. 48: 4581-4589.

15. Hagerman. A.E. and L.G. Butler. 1980. Condensed tannin purification and characterization of tanninassociated proteins. Journal of Agricultural and Food Chemistry, 28(5): 947-52.

16. Hashemzadeh Cigari, F., M. Khorvash, G. Ghorbani, E. Ghasemi, A. Taghizadeh, S. Kargar, and W. Yang. 2014. Interactive effects of molasses by homofermentative and heterofermentative inoculants on fermentation quality, nitrogen fractionation, nutritive value and aerobic stability of wilted alfalfa (Medicago sativa L) silage. Journal of Animal Physiology and Animal Nutrition, 98: 290-299.

17. Hernandez, F., P. Melgarejo, F.A. Tomas-Barberan and A. Artes. 1999. Evolution of juice anthocyanins during ripening of new selected pomegranate (Punica granatum) clones. European Food Research and Technology, 210: 39-42. 
18. Kyralan, M., M. Golukcu and H. Tokgoz. 2009. Oil and conjugated linolenic acid contents of seeds from important pomegranate cultivars (Punica granatum L.) grown in Turkey. Journal of the American Oil Chemist's Society, 86: 985-990.

19. Lansky, E., S. Shubert and I. Neeman. 1998. Pharmacological and therapeutical properties of pomegranate. Proceedings of $1^{\text {st }}$ International Symposium on Pomegranate, 231-235 pp., Orihuela, Spain.

20. Makkar, H.P.S. 2000. A laboratory manual for the FAO/IAEA co-ordinated research project on 'Use of Nuclear and Related techniques to Develop Simple Tannin Assays for Predicting and Improving the safety and Efficiency of Feeding Ruminants on Tanniniferous Tree Foliage'. Joint FAO/IAEA of Nuclear Techniques in Food and Agriculture. Animal Production and Health Sub-Progrmme, FAO/IAEA Working Document. IAEA, Vienna, Austria.

21. Makkar, H.P.S. 2003. Quantification of tannins in tree and shrub foliage: A laboratory manual. Food and Agriculture Organization of the United Nations/International Atomic Energy Agency. Kluwer Academic Publishers, Dordrecht, the Netherlands, $102 \mathrm{pp}$.

22. McDonald, P. 1981. The biochemistry of silage. John Wiley and Sons Ltd., Toronto, $226 \mathrm{pp}$.

23. Menke, K.H. L. Raab, A. Salewski, H. Steingass, D. Fritz and W. Schneider. 1979. The estimation of the digestibility and metabolizable energy content of ruminant feedigstuffs from the gas production when they are incubated with rumen liquor in vitro. Journal of Agricultural Science, 93: $217-222$.

24. Mirzaei Aghsaghali, A. and N. Maheri Sis. 2008a. Nutritive value of some agro-industrial by-products for ruminants. A review. World Journal of Zoology, 3(2): 40-46.

25. Mirzaei Aghsaghali, A., N. Maheri Sis, H. Mansouri, M.E. Razeghi, A. Mirza Aghazadeh, H. Cheraghi and A. Aghajanzadeh Golshani. 2011. Evaluating potential nutritive value of pomegranate processing by products for ruminants using in vitro gas production technique. ARPN Journal of Agricultural and Biological Science, 6(6): 45-51.

26. Ørskov, E. and I. McDonald. 1979. The estimation of protein degradability in the rumen from incubation measurements weighted according to rate of passage. The Journal of Agricultural Science, 92: 499-503.

27. Parseh, H., S. Hassanpour, Z. Emam Djome, A.S. Lavasani, H.Z. Mahmoodabady, M. CHabok, F. Morady, G.R. Cheshmali, J. Sarhadi and M. Hematian. 2011. Antimicrobial properties of Pomegranate (Punica granatum L.) as a Tannin rich Fruit: a review. Proceedings of the $1^{\text {th }}$ international and the 4th national congress on recycling of organic waste in agriculture, Isfahan, Iran. (In Persian).

28. Playne, M. and P. McDonald. 1966. The buffering constituents of herbage and of silage. Journal of the Science of Food and Agriculture, 17: 264-268.

29. Robertson, J. and M. Eastwood, 1981. A method to measure the water-holding properties of dietary fibre using suction pressure. British Journal of Nutrition, 46: 247-255.

30. Safaei, A.R., N.M. Torbatinejad, H. Mansouri and S. Zarehdaran. 2014. Effects of adding PolyEthylene-Glycol on methane production in rumen, digestion and metabolic energy of grape and gime pomaces. Research on Animal Production, 5: 83-95.

31. SAS. 2009. Version 9.1. Guide, STAT User's, SAS Institute Inc., Cary, NC.

32. Shabtay, A., H. Eitam, Y. Tadmor, A. Orlov, A. Meir, P. Weinberg, Z.G. Weinberg, Y. Chen, A.I. Brosh, I. Zhaki and Z. Kerem. 2008. Nutritive and antioxidative potential of fresh and stored pomegranate industrial byproduct as novel beef cattle feed. Journal of Agricultural and Food Chemistry, 56: 10063-10070.

33. Shahidi, F. and M. Naczk. 2004. Phenolics in food and nutraceuticals. CRC Press Book, US, 576 pp.

34. Silanikove, N., A. Perevolotsky and F.D. Provenza. 2001. Use of tannin binding chemicals to assay for tannins and their negative post-ingestive effects in ruminants. Animal Feed Science and Technology, 91: 69-81.

35. Sommart, K., D.S. Parker, P. Rowlinson and M. Wanapat. 2000. Fermentation characteristics and microbial protein synthesis in an in vitro system using cassava, rice straw and dried ruzi grass as substrates. Asian-Australasian Journal of Animal Sciences, 13: 1084-1093.

36. Taher Maddah, M., N. Maheri Sis, R. Salamatdoustnobar and A. Ahmadzadeh. 2012a. Comparing nutritive value of ensiled and dried pomegranate peels for ruminants using in vitro gas production technique. Annals of Biological Research, 3(4): 1942-1946.

37. Taher Maddah, M., N. Maheri Sis, R. Salamatdoustnobar and A. Ahmadzadeh. 2012b. Estimating fermentation characteristics and nutritive value of ensiled and dried pomegranate seeds for ruminants using in vitro gas production technique. Open Veterinary Journal, 2: 40-45.

38. Teimoury Chamebon, A., A. Teimori Yanesari, Y. Chashnidel and A. Gafary Sayadi. 2017. Study of chemical composition, quality and ruminal degradability parameters of silaged orange pulp with wheat straw and urea. Research on Animal Production, 8: 84-95.

39. Tsuzuki, T., Y. Kawakami, R. Abe, K. Nakagawa, K. Koba, J. Imamura, T. Iwata, I. Ikeda and T. Miyazawa. 2006. Conjugated linolenic acid is slowly absorbed in rat intestine, but quickly converted to conjugated linoleic acid. Journal of Nutrition, 136: 2153-2159.

40. Tzulker, R., I. Glazer, I. Bar-Ilan, D. Holland, M. Aviram and R. Amir. 2007. Antioxidant activity, polyphenol content and related compounds in different fruit juices and homogenates prepared from 29 different pomegranate accessions. Journal of Agricultural and Food Chemistry, 55: 9559-9570.

41. Van Soest, P.J., J. Robertson and B. Lewis. 1991. Methods for dietary fiber, neutral detergent fiber, and nonstarch polysaccharides in relation to animal nutrition. Journal of Dairy Science, 74: 35833597.

42. Viuda-Martos, M., J. Fernández-López and J. Pérez-Álvarez. 2010. Pomegranate and its many functional components as related to human health: a review. Comprehensive Reviews in Food Science and Food Safety, 9: 635-665. 


\title{
Evaluating Chemical Composition, Fatty Acid Profiles, Antioxidant Activity and Nutritive Value of Pomegranate By-Product using in Vitro Gas Production Technique
}

\author{
Simin Khorsandi ${ }^{1}$, Ahmad Riasi ${ }^{2}$ and Mohammad Khorvash ${ }^{3}$ \\ 1- PhD Student of Ruminant Nutrition, Isfahan University of Technology \\ 2- Associate Professor, Isfahan University of Technology (Corresponding Author: ariasi@cc.iut.ac.ir) \\ 3- Associate Professor, Isfahan University of Technology \\ Received: January 6, 2018 Accepted: August 28, 2018
}

\begin{abstract}
This study was conducted to determine the important nutritional properties of pomegranate by-product (seed and peel) for evaluating its potential in silage preparation compared to corn forage. At first, enough fresh pomegranate by-products were prepared from a fruit juice factory in the fall season. Then, the chemical composition, fatty acid profiles, phenolic compounds and gas production parameters were measured in dried pomegranate and corn forage samples. Also, the parameters of silage preparation were determined in fresh samples. The results showed that dry matter, crud protein, ether extract, neutral detergent fiber, acid detergent fiber and lignin content of pomegranate seed was higher $(\mathrm{P}<0.05)$ than peel. But, the water soluble carbohydrates and ash content of pomegranate seed was lower $(\mathrm{P}<0.05)$ than pomegranate peel. The water soluble carbohydrates in pomegranate peel was about two times more than corn forage. Moreover, pomegranate peel had high content of phenolic compounds $(6.17 \%)$, total tannin (4.5\%) and antioxidant activity (99.1\%). Pomegranate seed and peel had $88.3 \%$ and $77.9 \%$ unsaturated fatty acids, respectively and the punicic acid content of pomegranate seed and peel was $64.9 \%$ and $46.9 \%$ of total fatty acids, respectively. The gas production parameters showed that metabolizable energy, net energy for lactation, short chain fatty acid, and organic matter digestibility in pomegranate seed and peel were lower $(\mathrm{P}<0.05)$ than corn forage. The buffering capacity of the mixture of pomegranate seed and peel $(1: 4)$ was in the proper range for the preparation of silage. Water storage capacity in the mixture of pomegranate seed and peel was lower $(\mathrm{P}<0.05)$ than corn forage. It was concluded that the mixture of pomegranate seed and peel (1:4) has suitable chemical composition for preparation of silage, and due to the antioxidant activity and fatty acids profile, could be considered as a valuable feed ingredient in replacement with the part of ruminant's required silage.
\end{abstract}

Keywords: Agriculture by-product, Seed and peel of pomegranate, Phenolic compounds, Tannin, Essential Fatty acid, Antioxidant activity, Silage of pomegranate by-product 